\title{
Topological Character and Magnetism of the Dirac State in Mn-Doped $\mathrm{Bi}_{2} \mathrm{Te}_{3}$
}

\author{
J. Henk, ${ }^{1, *}$ M. Flieger, ${ }^{1}$ I. V. Maznichenko, ${ }^{1}$ I. Mertig, ${ }^{1,2}$ A. Ernst, ${ }^{2}$ S. V. Eremeev, ${ }^{3,4,5}$ and E. V. Chulkov ${ }^{5,6,7}$ \\ ${ }^{1}$ Institut für Physik, Universität Halle-Wittenberg, Von-Seckendorff-Platz 1, D-06120 Halle, Germany \\ ${ }^{2}$ Max-Planck-Institut für Mikrostrukturphysik, Weinberg 2, D-06120 Halle, Germany \\ ${ }^{3}$ Institute of Strength Physics and Materials Science, Prospekt Academicheskii 2/4, 634021 Tomsk, Russia \\ ${ }^{4}$ Tomsk State University, Prospekt Lenina 36, 634050, Tomsk, Russia \\ ${ }^{5}$ Donostia International Physics Center (DIPC), Paseo de Manuel Lardizabal, \\ 4, 20018 San Sebastián/Donostia, Basque Country, Spain \\ ${ }^{6}$ Departamento de Física de Materiales, Facultad de Ciencias Químicas, UPV/EHU, Apartado 1072, \\ 20080 San Sebastián, Basque Country, Spain \\ ${ }^{7}$ Centro de Física de Materiales, CFM-MPC, Centro Mixto CSIC-UPV/EHU, Apartado 1072, \\ 20080 San Sebastián/Donostia, Basque Country, Spain
}

(Received 10 April 2012; published 16 August 2012; publisher error corrected 7 September 2012)

First-principles and model calculations show that the Dirac surface state of the topological insulator $\mathrm{Bi}_{2} \mathrm{Te}_{3}$ survives upon moderate $\mathrm{Mn}$ doping of the surface layers but can lose its topological character as a function of magnetization direction. The dispersion depends considerably on the direction of the $\mathrm{Mn}$ magnetization: for perpendicular magnetization, a gap of $16 \mathrm{meV}$ opens up at the Dirac point; for in-plane magnetization, a tiny gap can be opened or closed in dependence on the magnetization azimuth. The ground state is ferromagnetic, with a critical temperature of $12 \mathrm{~K}$. The results provide a path towards a magnetic control of the topological character of the Dirac surface state and its consequences to spindependent transport properties.

DOI: 10.1103/PhysRevLett.109.076801

PACS numbers: 73.20.At, 71.70.Ej, 75.70.Rf

Topological insulators are a new class of materials with promising properties for both fundamental physics and technological applications for upcoming spinelectronic devices [1,2]. The topological character in three-dimensional topological insulators, such as $\mathrm{Bi}_{2} \mathrm{Se}_{3}$ and $\mathrm{Bi}_{2} \mathrm{Te}_{3}$, manifests itself by a linearly dispersed surface state that bridges the bulk band gap. This Dirac surface state is topologically protected, meaning that it is robust against perturbations that maintain time-reversal symmetry (e.g., surface chemical disorder [3]). This raised a question on the robustness of these states against doping by magnetic impurities [4]. If time-reversal symmetry is broken due to magnetic order, the topological character can be strongly modified [5], as was demonstrated for surfacealloyed $\mathrm{Bi}_{2-x} \mathrm{Mn}_{x} \mathrm{Se}_{3}$ [1]. The unique spin texture of the Dirac surface state can cause the quantum spin-Hall effect [6-8], as it has been shown for $\mathrm{HgTe}$ quantum wells [9] or to a magnetic monopole [10]. For magnetically doped topological insulators, a quantized anomalous Hall effect has been predicted [11].

In this Letter, we provide strong support by a theoretical first-principles investigation that the surface state of the topological insulator $\mathrm{Bi}_{2} \mathrm{Te}_{3}$ doped with $\mathrm{Mn}$ in the topmost quintuple layer "survives" but with modified dispersion relation and spin texture. A striking feature is that the band structure close to the Dirac point in $\mathrm{Bi}_{2-x} \mathrm{Mn}_{x} \mathrm{Te}_{3}$ depends on the magnetization direction: a band gap of $16 \mathrm{meV}$ opens up for perpendicular magnetization, but for in-plane magnetization a tiny gap can be opened or closed in dependence on the magnetization's azimuth, a finding that complies fully with an extended Fu model [12]. This dependence of the Dirac state's topological character on the magnetization direction has consequences for spindependent transport, which now can be controlled by an external magnetic field. We also discuss magnetic properties (e.g., magnetic order, magnetic moments, and critical temperature).

$\mathrm{Bi}_{2} \mathrm{Te}_{3}$ is assembled by quintuple layers (QLs) that comprise five hexagonal layers, i.e., two atomic Bi layers separated by atomic Te layers (Fig. 1). If moderately doped with $\mathrm{Mn}$ [13], $\mathrm{Bi}_{2} \mathrm{Te}_{3}$ forms a substitutional surface alloy in which $\mathrm{Bi}$ atoms are randomly replaced by $\mathrm{Mn}$ atoms [4].

First-principles calculations were performed within the local spin-density approximation to density functional theory, with Perdew-Wang exchange-correlation functional [14]. The electronic and magnetic properties were obtained by scalar-relativistic and relativistic Korringa-KohnRostoker methods $[15,16]$.

To mimic experiments [4], Mn-doped samples were treated as substitutional binary alloys in which the $\mathrm{Bi}$ atoms in the topmost QL are replaced by effective $\mathrm{Bi}_{0.9} \mathrm{Mn}_{0.1}$ atoms. The disorder is treated within the coherent potential approximation [17,18].

Detailed information on the spin-resolved electronic structure is obtained from the site- and momentumresolved Green function $G_{i i}^{+}\left(E, \vec{k}_{\|}\right)$of the system $[i$ site index, $\vec{k}_{\|}=\left(k_{x}, k_{y}\right)$ wave vector parallel to the layers]. The Bloch spectral density is given by 
(b) side view

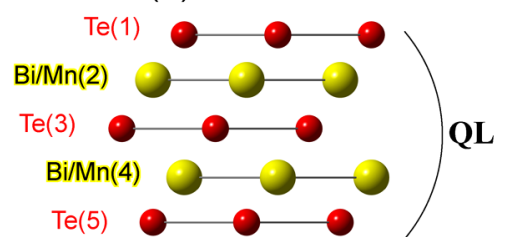

(a) top view
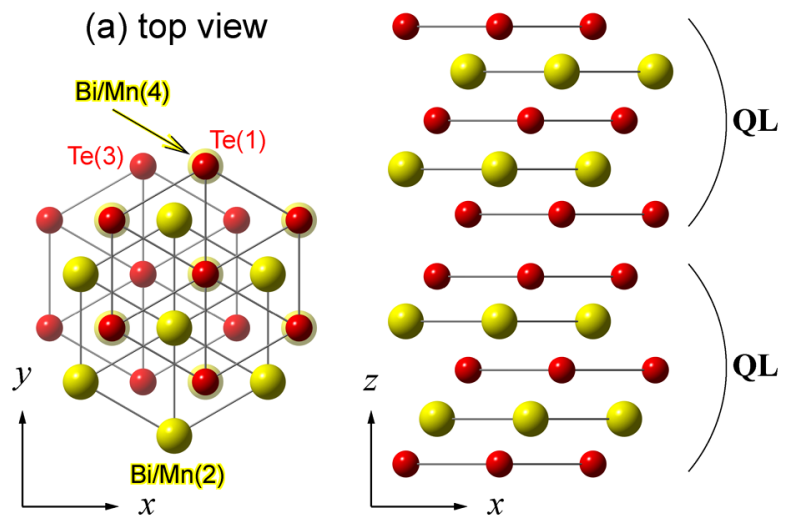

FIG. 1 (color online). (111) surface of $\mathrm{Bi}_{2-x} \mathrm{Mn}_{x} \mathrm{Te}_{3}$. (a) Top view. $\mathrm{Bi}$ and $\mathrm{Te}$ atoms are represented by yellow (light) and red (dark) spheres, respectively, and form hexagonal layers. Numbers in parentheses give the layer index, the topmost layer labeled (1). (b) Side view. A stack of three quintuple layers is shown. Only the topmost QL is substitutionally alloyed: pure Bi layers (2) and (4) are replaced by $\mathrm{Bi}_{0.9} \mathrm{Mn}_{0.1}$ layers.

$$
N_{i}\left(E, \vec{k}_{\|}\right)=-\frac{1}{\pi} \operatorname{Im} \operatorname{Tr} G_{i i}^{+}\left(E, \vec{k}_{\|}\right),
$$

where the trace is over the site $i$.

For an analytical description of the Dirac state, we extend Fu's model Hamilton operator [12] by magnetism,

$$
\hat{H} \equiv \hat{H}_{\mathrm{ph}}+\hat{H}_{\mathrm{soc}}+\hat{H}_{\mathrm{warp}}+\hat{H}_{\text {magn }} .
$$

The free-electron dispersion $\hat{H}_{\mathrm{ph}}=E_{0}\left(\vec{k}_{\|}\right)$breaks the particle-hole symmetry. $\hat{H}_{\mathrm{soc}}=v_{k}\left(k_{x} \sigma_{y}-k_{y} \sigma_{x}\right)$ accounts for the Rashba-Bychkov spin-orbit coupling ( $v_{k}$ Fermi velocity) and results in in-plane spin components that are perpendicular to the momentum $\vec{k}_{\|} . \hat{H}_{\text {warp }}=$ $\frac{\lambda}{2}\left(k_{+}^{3}+k_{-}^{3}\right) \sigma_{z}$ introduces the hexagonal warping $\left(k_{ \pm} \equiv\right.$ $k_{x} \pm i k_{y}$ ) and leads to out-of-plane spin components. $\hat{H}_{\text {magn }}=-J n_{S} \vec{S} \cdot \vec{\sigma}$ couples the electron's spin $\vec{\sigma}$ to localized spins $\vec{S}$ that are due to the Mn impurities, with density $n_{S} . J$ is the effective exchange constant, and $\vec{\sigma}=$ $\left(\sigma_{x}, \sigma_{y}, \sigma_{z}\right)$ is the vector of Pauli matrices [19]. We solve the Schrödinger equation for this Hamilton operator both analytically and numerically, taking $v_{k}, \lambda$, and $J n_{S}|S|$ as parameters adjusted to agree well with the ab initio results. The purpose of the model calculations is to provide a tool for understanding the dispersion of the Dirac state that is obtained from the first-principles calculations. The detailed coupling mechanism of the Dirac electron's spin with the localized Mn spins $\vec{S}$ is beyond the scope of the present investigation [20-22].

Ferromagnetism of moderately doped $\mathrm{Bi}_{2-x} \mathrm{Mn}_{x} \mathrm{Te}_{3}$ $(x=0.2)$ is supported by our first-principles calculations. The calculated local magnetic moments are $4.58 \mu_{\mathrm{B}}$ (second layer) and $4.52 \mu_{\mathrm{B}}$ (fourth layer). Mapping of the Korringa-Kohn-Rostoker results onto a classical Heisenberg model, using the magnetic force theorem for the substitutional alloy, delivers also the exchange constants $J_{i j}$ [23] (for details see the Supplemental Material [24]). In contrast to Mn's tendency to antiferromagnetic order in many materials, we find a ferromagnetic ground state, with a Curie temperature of about $12 \mathrm{~K}$ (experiment: 9-12 $\mathrm{K}$ for $x=0.18$ [4]). The collinear alignment of the localized Mn spins $\vec{S}$ can thus be characterized by a magnetization $\vec{M}$. For the purpose of this Letter, only the direction of $\vec{M}$ matters.

In the following, we discuss results for samples with $\vec{M} \| \vec{x}$ (in-plane, within the $x z$ mirror plane of the (111) surface; Fig. 1) and $\vec{M} \| \vec{z}$ (normal to the surface; out-ofplane magnetic anisotropy was found experimentally by Hor et al. [4]). We do not show first-principles data for $\vec{M} \| \vec{y}$; these are very similar to those for $\vec{M} \| \vec{x}$, although they show a tiny band gap at the Dirac point (discussed below) and, thus, reveal a topologically different character of the Dirac state. Strictly speaking, the surface state of $\mathrm{Bi}_{2-x} \mathrm{Mn}_{x} \mathrm{Te}_{3}$ is no longer a Dirac state (characterized by linear dispersion and a Dirac cone without band gap); we nevertheless call it a Dirac state to avoid unnecessary and perhaps confusing nomenclature.

The Dirac surface state of $\mathrm{Bi}_{2} \mathrm{Te}_{3}$ shows up as a single band of conical shape crossing the fundamental bulk band gap (Fig. 2 and Refs. [25,26]). It disperses linearly along $\vec{k}_{x}$ $[\bar{K}-\bar{\Gamma}-\bar{K}$, Fig. 2(a)] but displays a "curved" dispersion along $\vec{k}_{y}[\bar{M}-\bar{\Gamma}-\bar{M}$, Fig. 2(b)], which indicates warping [27]. The blurred regions mainly below the Fermi energy $E_{\mathrm{F}}$ in Fig. 2 are attributed to the surface part of bulk electronic states.

The surface state of $\mathrm{Bi}_{2} \mathrm{Te}_{3}$ survives $\mathrm{Mn}$ doping in the topmost QL. It shows linear dispersion, in particular along $\vec{k}_{x}$ (Figs. 2(c) and 2(e); a similar result was found by Niu et al. [27]), but the curved dispersion along $\vec{k}_{y}$ [Figs. 2(d) and 2(f)] is changed to an almost linear one. The density becomes weak and blurred with increasing energy, due to disorder and (at higher energies) hybridization with bulk electronic states. Furthermore, the surface state's spectral density in $\mathrm{Bi}_{2-x} \mathrm{Mn}_{x} \mathrm{Te}_{3}$ has a clear maximum that shows up as dark spot in Figs. 2(c)-2(f), in contrast to $\mathrm{Bi}_{2} \mathrm{Te}_{3}$. These findings compare nicely with experimental photoemission data for $\mathrm{Bi}_{2-x} \mathrm{Mn}_{x} \mathrm{Te}_{3}$ with $18 \%$ Mn doping [4].

Constant-energy cuts through the dispersion relation $E\left(\vec{k}_{\|}\right)$are circular at energies close to the Dirac point. The surface state's spin $\vec{s}$ is perpendicular to the surface-parallel 

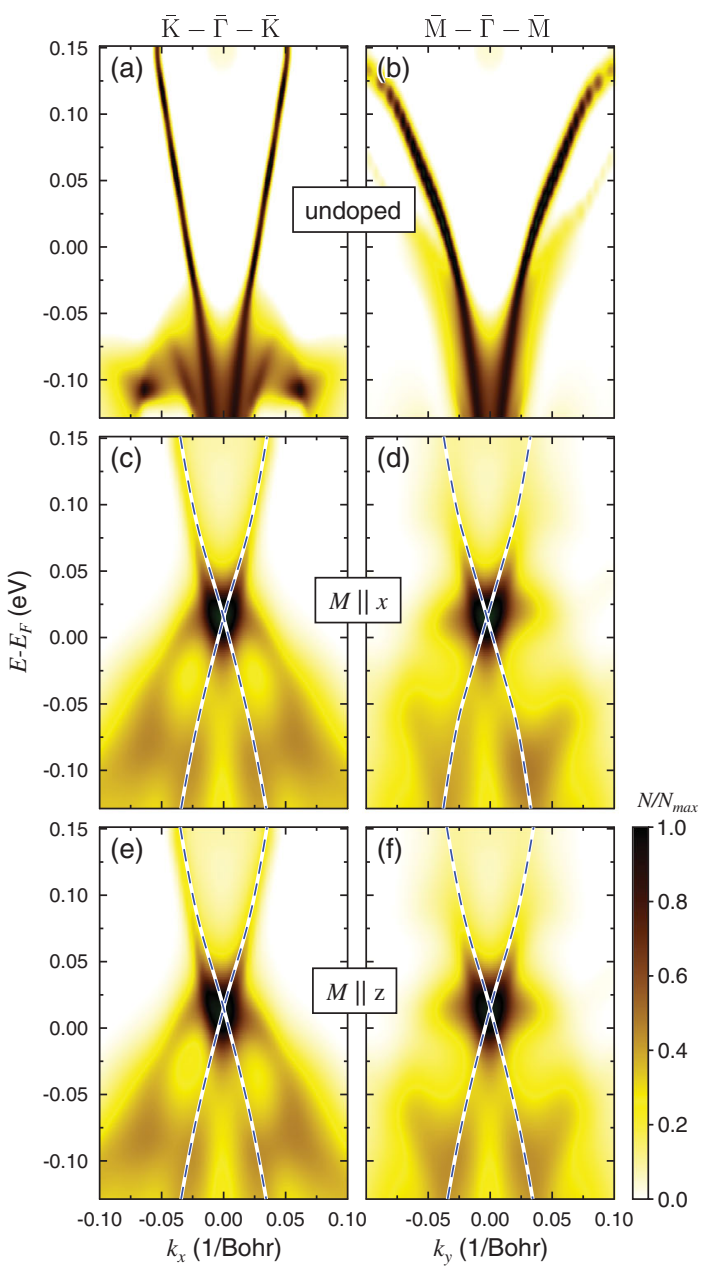

FIG. 2 (color). Dispersions $\left(E, \vec{k}_{\|}\right)$of the Dirac surface state in $\mathrm{Bi}_{2} \mathrm{Te}_{3}$ [undoped; (a) and (b)] and $\mathrm{Bi}_{2-x} \mathrm{Mn}_{x} \mathrm{Te}_{3}$ [doped; $10 \%$ Mn with magnetization $\vec{M}$ parallel to $\vec{x}$ (c), (d) and $\vec{z}$ (e), (f)] for electron momenta $\vec{k}=\left(k_{x}, k_{y}\right)$ along $\vec{k}_{x}(\bar{K}-\bar{\Gamma}-\bar{K}$ in the twodimensional Brillouin zone; left column) and $\vec{k}_{y}(\bar{M}-\bar{\Gamma}-\bar{M}$; right column). The linear color scale presents the spectral weight of a top-layer Te site, normalized in each panel. The Fermi level is at $0 \mathrm{eV}$. Dashed lines in (c)-(f) highlight the dispersion of the Dirac surface state.

component of the electron momentum $\hbar \vec{k}_{\|}$[28]. At greater energy distances from the Dirac point, these momentum distributions become hexagonally shaped (Fig. 3(b); cf. Fig. 13 in Ref. [4]) and eventually turn into a "snowflake" [Fig. 3(a)].

For the Mn-doped sample with magnetization along $\vec{x}$ [Figs. 3(c) and 3(d)], the momentum distributions are blurred due to disorder. Further, hybridization with bulk states results in six raylike features [Fig. 3(c)] that correspond to the cusps in the $\mathrm{Bi}_{2} \mathrm{Te}_{3}$ momentum distribution [Fig. 3(a)]. For the magnetization being along $\vec{z}$ (out-ofplane), the contour for the largest energies is slightly smaller [Fig. 3(f)], indicating a different dispersion relation than that for $\vec{M} \| \vec{x}$. A closer look reveals that the
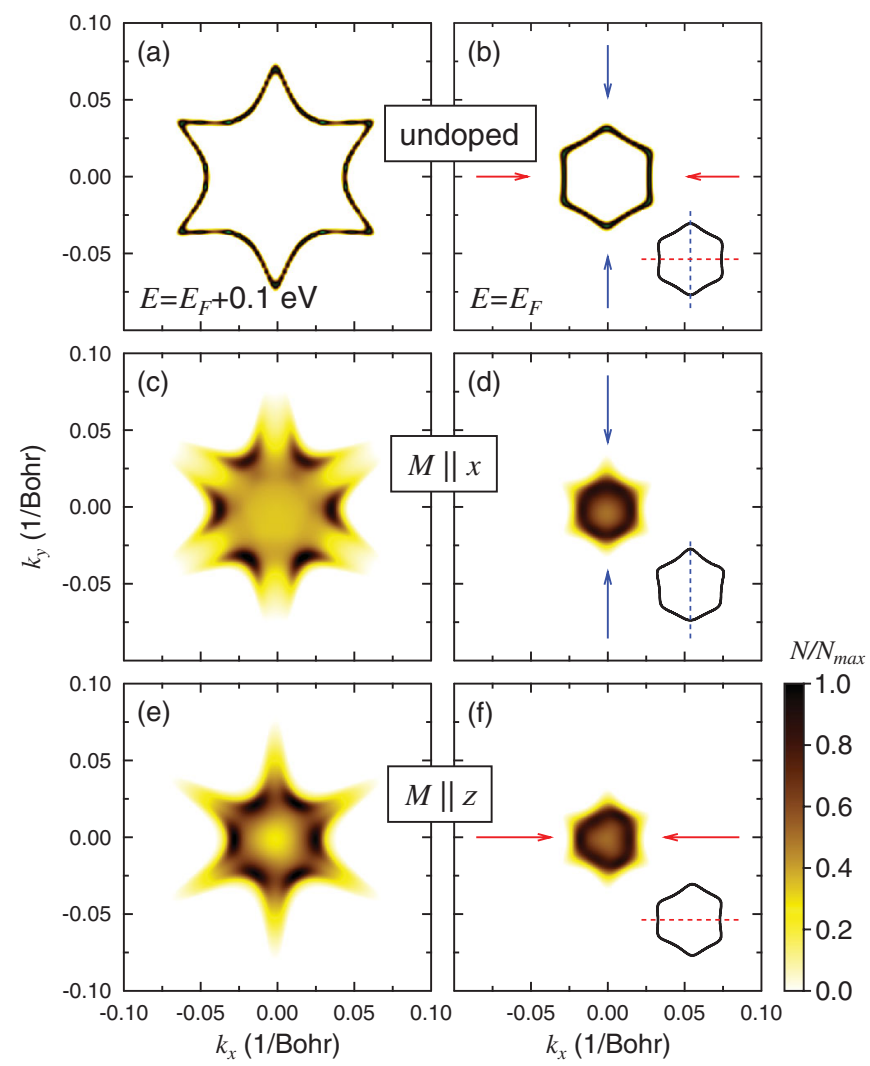

FIG. 3 (color). Constant-energy contours of the Dirac-state dispersions in $\mathrm{Bi}_{2} \mathrm{Te}_{3}$ [undoped; (a) and (b)] and $\mathrm{Bi}_{2-x} \mathrm{Mn}_{x} \mathrm{Te}_{3}$ [doped; (c)-(f)]. The energies are the Fermi energy $E_{\mathrm{F}}$ (right column) and $E_{\mathrm{F}}+0.1 \mathrm{eV}$ (left column). The linear color scale presents the spectral weight of a top-layer Te site, normalized in each panel. Blue and red arrows in the right column indicate mirror axes of the constant-energy contours. Further, insets sketch contours at the Brillouin zone center obtained from the model Hamiltonian in (2).

contours are asymmetric, which is most clearly seen at the smaller energy [Figs. 3(d) and 3(f)].

To clarify the magnetic origin of these asymmetries, we added a magnetic term to Fu's analytical model [12], $\hat{H}_{\text {magn }}$ in Eq. (2). In all cases, the first-principles contours are nicely reproduced [insets in Figs. 3(b), 3(d), and 3(f)]. For $\vec{M} \| \vec{x}$ [Fig. 3(d)], the $y z$ mirror symmetry is maintained whereas the $x z$ mirror symmetry is broken; this is typical for Rashba-Bychkov systems with in-plane magnetization [29]. For $\vec{M} \| \vec{z}$ [Fig. 3(f)], the $x z$ symmetry is maintained but the $y z$ symmetry is broken, yielding a threefold rotational symmetry. A discussion of Dirac-state dispersion and magnetization direction by means of the extended $\mathrm{Fu}$ model is provided in the Supplemental Material [24].

The topological character of the Dirac state is closely related to an exchange-mediated band gap at the Dirac point of $\mathrm{Bi}_{2-x} \mathrm{Mn}_{x} \mathrm{Te}_{3}$ [Figs. 4(b) and 4(c)]. We calculated the spectral density on a very fine energy mesh at the Brillouin zone center $\left(\vec{k}_{\|}=0\right)$ and find two peaks with opposite spin character $S_{z} \equiv N_{i \uparrow}-N_{i \downarrow}$ for $\vec{M} \| \vec{z}$ [Fig. 4(b)], a clear 


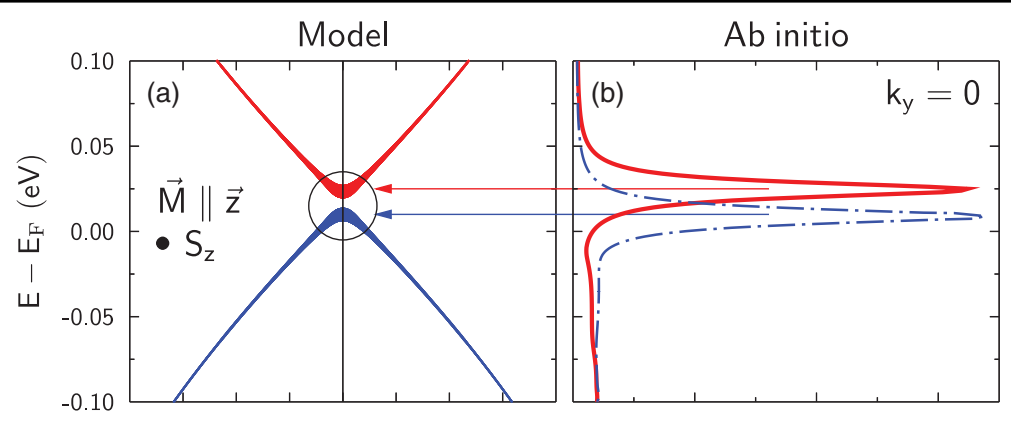

(c)
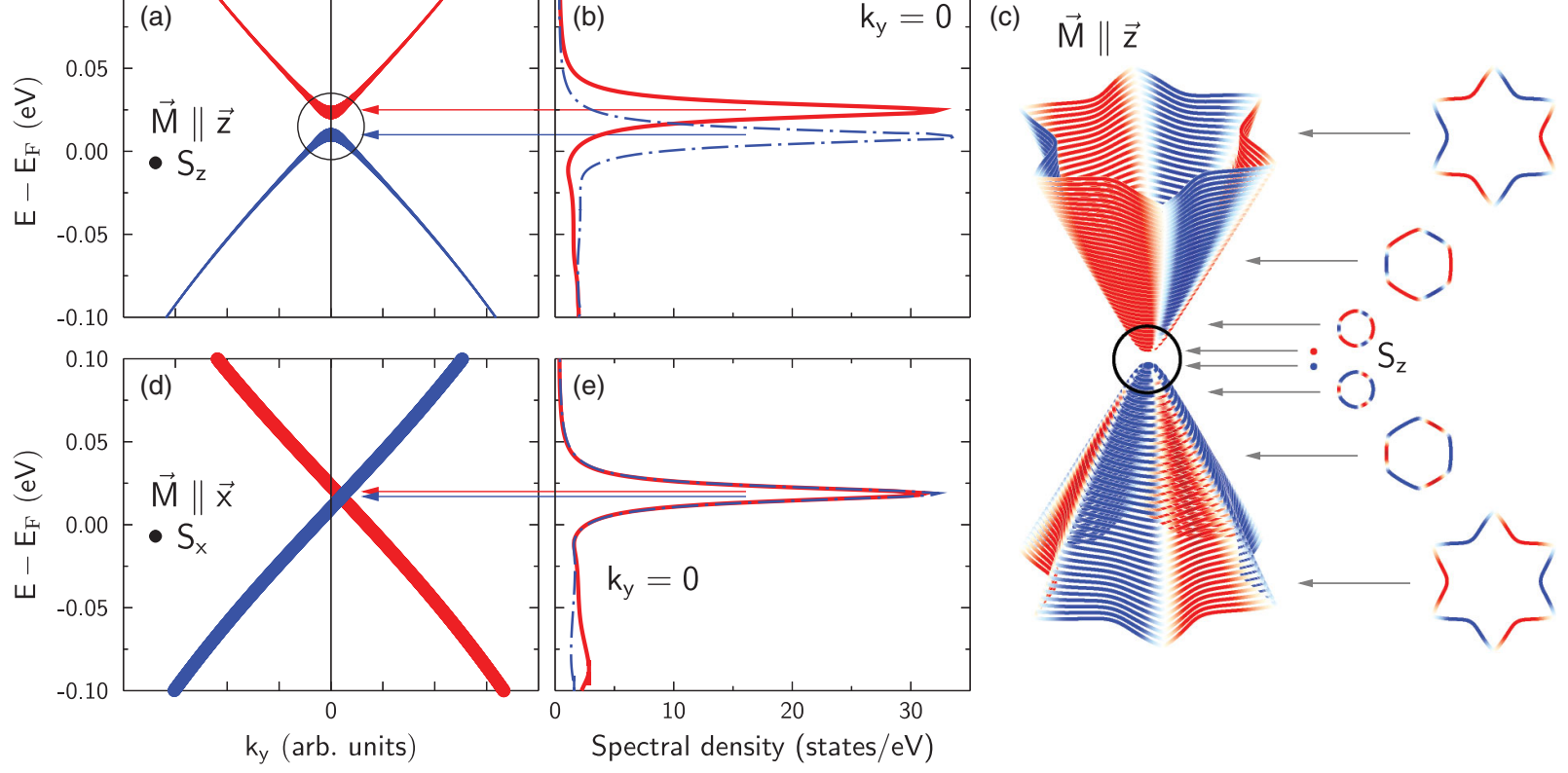

FIG. 4 (color). Spin-resolved electronic structure at the Dirac point of $\mathrm{Bi}_{2-x} \mathrm{Mn}_{x} \mathrm{Te}_{3}$, for $\vec{M} \| \vec{z}$ (a)-(c) and $\vec{M} \| \vec{x}$ (d), (e). (a) Schematic surface-state band structure along $k_{y}$, obtained from the model in Eq. (2) (model). The line thickness is proportional to the absolute degree of $S_{z}$, whereas its color represents the sign (blue negative, white zero, red positive). The black circle highlights the band gap. (b) Spectral density at the Te top site computed from first principles (ab initio) at $k_{y}=0$. The two peaks are attributed to the band edges (cf. the horizontal arrows). (c) Sketch of dispersion and constant energy contours in a large energy range, with the band gap highlighted by a black circle. (d), (e) Analogous data for $\vec{M} \| \vec{x}$ and $S_{x}$, for which there is no band gap but a shift of the Dirac point off the Brillouin zone center. The model parameters used in (a) and (d) are identical.

indication of a band gap of about $16 \mathrm{meV}$ (although shown only for the Te top site, the gap opens up in the entire QL; the spectral density of the Te site in that energy range is greater by a factor of about 9 than that of the other sites). This finding is fully in line with the model calculation, as indicated by the arrows in Fig. 4(a): the negatively polarized (red) peak is associated with the maximum of the lower (red) band while the positively polarized (blue) peak is attributed to the band minimum of the upper (blue) band. In the model calculations, the Dirac state is mainly spinpolarized in-plane far from the band gap but the perpendicular component $S_{z}$ dominates at $\vec{k}=0$ [cf. the increased line width at $k_{y}=0$ in Fig. 4(a)].

For $\vec{M} \| \vec{x}$, the energy scan at $k_{y}=0$ crosses the spinpolarized bands, giving rise to two maxima in the spectral density [Fig. 4(e)]. The two peaks coincide almost completely but are oppositely spin-polarized along $\vec{x}$. In the respective model calculation [Fig. 4(d)], there is no band gap but the Dirac point is shifted off the Brillouin zone center. This displacement appears as asymmetric spectraldensity contours in Figs. 2(d) and 3(d).

In the first-principles calculation for magnetization along $\vec{y}$ (not shown), we find a result very similar to that for $\vec{M} \| \vec{x}$. In particular, there is no apparent band gap. More precisely, the gap width is less than $1 \mathrm{meV}$, that is, the energy-step width in the calculations. This finding is supported by our model in which a band gap of about $0.25 \mathrm{meV}$ shows up for $\vec{M} \| \vec{y}$, in contrast to the band gap of $16 \mathrm{meV}$ for $\vec{M} \| \vec{z}$ [Figs. 4(a) and 4(b)]. This agreement corroborates that Fu's model fully captures essential properties of the Dirac state although it deals with an idealized system. In the model, for example, the Dirac electron is strictly confined to the $x y$ plane and its degree of spin polarization is $100 \%$. In the firstprinciples calculations, however, the Dirac state of $\mathrm{Bi}_{2} \mathrm{Te}_{3}$ shows a complicated spin and orbital composition within the entire outermost QL, with the consequence that its degree of spin polarization is reduced to less than $45 \%$ (the latter finding is fully in line with earlier theoretical results [30]).

Since the magnetic term in the model Hamiltonian is equivalent to a Zeeman term that couples the Dirac state's spin to an external magnetic field, one could speculate that the findings reported here could show up in a magnetically undoped topological insulator but in an external magnetic field. The effect of an external magnetic field on the Dirac state of a topological insulator has been addressed mainly with respect to transport properties, in particular to a quantized Hall effect (see for example Refs. [31-35]). Kharitonov recently showed by a model calculation [36] that the size of the band gap in the Dirac state is about 10 times larger for perpendicular direction of the external 
magnetic field (about $2.5 \mathrm{meV}$ at $1 \mathrm{~T}$ ) than that for an in-plane direction. These findings agree qualitatively with ours on Mn-doped $\mathrm{Bi}_{2} \mathrm{Te}_{3}$.

In summary, we demonstrated by first-principles calculations that Mn doping of the $\mathrm{Bi}_{2} \mathrm{Te}_{3}$ surface layers causes ferromagnetic order. As a consequence, the Dirac surface state is gapped by $16 \mathrm{meV}$ for perpendicular magnetic anisotropy. For in-plane magnetic anisotropy, the Dirac point is shifted perpendicular to the magnetization direction; if the magnetization lies within a mirror plane of the surface, a tiny band gap opens up (about $0.25 \mathrm{meV}$ ), in contrast to the case when the magnetization is perpendicular to the mirror plane in which a gap does not open up. These findings on the band-gap behavior of Mn-doped $\mathrm{Bi}_{2} \mathrm{Te}_{3}$ establish a tool for magnetic control of the spindependent transport at the surface of a topological insulator. By switching the magnetization direction from in-plane to out-of-plane by an external magnetic field, the band gap at the Dirac point can be opened and vice versa. Consequently, the quantum spin Hall effect $[9,37]$ can be switched off and on.

*Corresponding author. juergen.henk@physik.uni-halle.de

[1] Y. L. Chen, J.-H. Chu, J. G. Analytis, Z. K. Liu, K. Igarashi, H.-H. Kuo, X. L. Qi, S. K. Mo, R. G. Moore, D. H. Lu, M. Hashimoto, T. Sasagawa, S. C. Zhang, I. R. Fisher, Z. Hussain, and Z. X. Shen, Science 329, 659 (2010).

[2] M. Franz, Science 329, 639 (2010).

[3] L. A. Wray, S.-Y. Xu, Y. Xia, D. Hsieh, A. V. Fedorov, Y. S. Hor, R. J. Cava, A. Bansil, H. Lin, and M. Z. Hasan, Nature Phys. 7, 32 (2010).

[4] Y. S. Hor, P. Roushan, H. Beidenkopf, J. Seo, D. Qu, J. G. Checkelsky, L. A. Wray, D. Hsieh, Y. Xia, S.-Y. Xu, D. Qian, M.Z. Hasan, N. P. Ong, A. Yazdani, and R. J. Cava, Phys. Rev. B 81, 195203 (2010).

[5] Q. Liu, C.-X. Liu, C. Xu, X.-L. Qi, and S.-C. Zhang, Phys. Rev. Lett. 102, 156603 (2009).

[6] J. E. Hirsch, Phys. Rev. Lett. 83, 1834 (1999).

[7] J. Sinova, D. Culcer, Q. Niu, N. A. Sinitsyn, T. Jungwirth, and A. H. MacDonald, Phys. Rev. Lett. 92, 126603 (2004).

[8] T. Kimura, Y. Otani, T. Sato, S. Takahashi, and S. Maekawa, Phys. Rev. Lett. 98, 156601 (2007).

[9] M. König, S. Wiedmann, C. Brüne, A. Roth, H. Buhmann, L. W. Molenkamp, X.-L. Qi, and S.-C. Zhang, Science 318, 766 (2007).

[10] X.-L. Qi, R. Li, J. Zang, and S.-C. Zhang, Science 323, 1184 (2009).

[11] R. Yu, W. Zhang, H.-J. Zhang, S.-C. Zhang, X. Dai, and Z. Fang, Science 329, 61 (2010).

[12] L. Fu, Phys. Rev. Lett. 103, 266801 (2009).
[13] D. Hsieh, Y. Xia, L. Wray, D. Quian, A. Pal, J. H. Dil, J. Osterwalder, F. Meier, G. Bihlmayer, C.L. Kane, Y.S. Hor, R. J. Cava, and M. Z. Hasan, Science 323, 919 (2009).

[14] J. P. Perdew and Y. Wang, Phys. Rev. B 45, 13244 (1992).

[15] J. Henk, in Handbook of Thin Film Materials, edited by H.S. Nalwa (Academic, New York, 2002), Vol. 2, Chap. 10, p. 479.

[16] Electron Scattering in Solid Matter, edited by J. Zabloudil, R. Hammerling, L. Szunyogh, and P. Weinberger (Springer, Berlin, 2005).

[17] P. J. Durham, B. L. Gyorffy, and A. J. Pindor, J. Phys. F 10, 661 (1980).

[18] A. Ernst, L. M. Sandratskii, M. Bouhassoune, J. Henk, and M. Lüders, Phys. Rev. Lett. 95, 237207 (2005).

[19] P. Strange, Relativistic Quantum Mechanics: With Applications in Condensed Matter and Atomic Physics (Cambridge University Press, Cambridge, England, 1998).

[20] T. Dietl, A. Haury, and Y. M. d'Aubigné, Phys. Rev. B 55, R3347 (1997).

[21] R. Skomski, J. Zhou, J. Zhang, and D. J. Sellmyer, J. Appl. Phys. 99, 08D504 (2006).

[22] D. A. Abanin and D. A. Pesin, Phys. Rev. Lett. 106, 136802 (2011).

[23] A. I. Liechtenstein, M. I. Katsnelson, V. P. Antropov, and V. A. Gubanov, J. Magn. Magn. Mater. 67, 65 (1987).

[24] See Supplemental Material at http://link.aps.org/ supplemental/10.1103/PhysRevLett.109.076801 for details on Dirac-state dispersion and magnetization direction as well as on magnetic properties.

[25] H. Zhang, C.-X. Liu, X.-L. Qi, Z. Fang, and S.-C. Zhang, Nature Phys. 5, 438 (2009).

[26] S. V. Eremeev, Y. M. Koroteev, and E. V. Chulkov, JETP Lett. 91, 387 (2010).

[27] C. Niu, Y. Dai, M. Guo, W. Wei, Y. Ma, and B. Huang, Appl. Phys. Lett. 98, 252502 (2011).

[28] Y. A. Bychkov and E. I. Rashba, J. Phys. C 17, 6039 (1984).

[29] O. Krupin, G. Bihlmayer, K. Starke, S. Gorovikov, J.E. Prieto, K. Döbrich, S. Blügel, and G. Kaindl, Phys. Rev. B 71, 201403(R) (2005).

[30] O. V. Yazyev, J.E. Moore, and S. G. Louie, Phys. Rev. Lett. 105, 266806 (2010).

[31] A. A. Zyuzin, M. D. Hook, and A. A. Burkov, Phys. Rev. B 83, 245428 (2011).

[32] A. A. Zyuzin and A. A. Burkov, Phys. Rev. B 83, 195413 (2011).

[33] Y.-Y. Zhang, X.-R. Wang, and X. C. Xie, J. Phys. Condens. Matter 24, 015004 (2012).

[34] V. M. Apalkov and T. Chakraborty, Phys. Rev. Lett. 107, 186801 (2011).

[35] O. A. Tretiakov, A. Abanov, and J. Sinova, J. Appl. Phys. 111, 07E319 (2012).

[36] M. Kharitonov, arXiv:1004.0194v1.

[37] A. Roth, C. Brüne, H. Buhmann, L. W. Molenkamp, J. Maciejko, X.-L. Qi, and S.-C. Zhang, Science 325, 294 (2009). 